\title{
Perspective d'ensemble sur les pénuries de médicaments des dernières décennies
}

\author{
par James L Mann
}

$\mathrm{L}$ a pharmacie hospitalière a une relation établie de longue date avec l'industrie pharmaceutique. À la fin des années 60 et dans les années 70, les pharmaciens d'hôpitaux ont été les promoteurs de l'établissement des groupements d'achats, mettant ainsi en commun le pouvoir d'achat d'établissements individuels au profit de groupes d'hôpitaux plus importants, mais tout de même facilement gérables. Les groupements d'achats ont développé des stratégies fiables pour établir les quantités de chaque produit pharmaceutique qu'achetait chaque groupe. Les services de pharmacie hospitalière faisaient pour leur part le suivi de la rotation des stocks et réduisaient les coûts de possession, avant même que cette méthode ne devienne la norme dans l'industrie de la santé. Les négociations avec l'industrie pharmaceutique reposaient sur une relation positive avec les fournisseurs basée non seulement sur le prix, mais sur de nombreux autres facteurs. Par exemple, les décisions d'achat pouvaient dépendre de la volonté du fournisseur d'adhérer à des normes d'emballage et d'étiquetage, comme celles développées par la Société canadienne des pharmaciens d'hôpitaux (SCPH) ${ }^{1}$, et de ses antécédents à composer avec les pénuries de médicaments et d'autres restrictions. Les groupements d'achats ont évolué et se sont améliorés grâce à l'engagement des pharmaciens qui se réunissaient avec leurs collègues de deux à trois fois par année pour définir leurs besoins et leurs préférences. Les pharmacies d'hôpitaux obtenaient de l'industrie des taux de couverture de $98 \%$ à $99 \%$, des livraisons ponctuelles et des représentants désireux et capables de s'adapter à des situations uniques à un hôpital en particulier.

Les gestionnaires des pharmacies d'hôpitaux justifiaient aux administrateurs de leur établissements les services d'additifs aux solutés, qui débouchaient sur des contrats avec les fabricants qui pouvaient fournir les produits pour faciliter le travail des pharmaciens d'hôpitaux et assurer un système considérablement plus sécuritaire pour les patients. Nous soutenions les activités des leaders de l'industrie en accordant aux joueurs novateurs une part du marché, plutôt que de permettre aux fournisseurs établis d'écarter les nouveaux joueurs en abaissant leurs prix. Les fournisseurs savaient que les groupements d'achats négociaient de bonne foi; ils savaient que, faute de circonstances imprévisibles, les pharmacies d'hôpitaux honoreraient leurs engagements d'achat.

Aujourd'hui, la plupart des hôpitaux et des régies régionales de la santé se sont affiliés à d'importantes entreprises d'achats groupés pour négocier des contrats " au nom des " pharmacies d'hôpitaux, groupements qui n'ont qu'une compréhension limitée de la nature complexe de la distribution des médicaments en établissements de santé. De nombreux représentants de l'industrie ont été éliminés ou on leur a assigné la responsabilité d'un nombre d'hôpitaux si important qu'il leur est impossible de développer une relation d'affaires avec chacun. Le service en a donc souffert. Cette tendance est particulièrement notable pour ce qui est des taux de couverture des commandes quotidiennes, qui ont énormément chuté, approchant souvent $80 \%$. La profession se trouve donc à devoir fournir des médicaments qui ne sont pas des agents de premier choix, des produits potentiellement moins efficaces ou plus toxiques et habituellement plus chers. Les pénuries de médicaments consomment aussi d'immenses ressources humaines de pharmacie, ce qui restreint la prestation des soins directs aux patients. Récemment, Sellinger ${ }^{2}$ a présenté un sommaire des résultats d'un sondage mené par la SCPH, l'Association des pharmaciens du Canada et l'Association médicale canadienne visant à déterminer les répercussions des pénuries de médicaments sur les pharmaciens, les médecins et les patients. Les pharmaciens d'hôpitaux qui ont répondu au sondage ont déclaré passer beaucoup de temps à composer avec ces pénuries, notamment à communiquer avec les acteurs clés, à rationner les médicaments, à préparer des médicaments à partir d'une concentration ou d'une forme pharmaceutique différente d'un même médicament, à changer de voie d'administration ou à recourir à un autre médicament ne 
faisant pas l'objet d'une pénurie ${ }^{2}$. D'autres inquiétudes concernant la sécurité des patients ont été soulevées, comme la substitution des médicaments qui requiert des modifications aux bibliothèques de médicaments utilisées par les technologies en « aval », comme les pompes à perfusion intelligentes ${ }^{3}$.

Vaillancourt ${ }^{4}$ a reconnu le besoin de maintenir une saine concurrence dans le marché canadien des médicaments afin de consolider la chaîne d'approvisionnement en médicaments essentiels et a suggéré de répartir les contrats entre deux ou trois fournisseurs afin de garantir que plus d'un fabricant soit en mesure de maintenir la chaîne de production. Nous devons demander aux groupements d'achats de favoriser les contrats multifournisseurs pour la majorité des produits, comme élément fondamental de la chaîne d'approvisionnement en médicaments. Dans le même contexte, "Les fournisseurs doivent être prêts à présenter une offre concurrentielle, même s'ils n'obtiennent pas la totalité du contrat, et les hôpitaux doivent être prêts pour leur part à payer plus pour une fraction du contrat ${ }^{4}$ ».

Un autre enjeu est comment obliger l'industrie pharmaceutique à rendre des comptes directement aux groupements d'achats ou aux hôpitaux que ces groupements représentent. Quels sont les principes de gestion de la qualité dictés aux fournisseurs dans les contrats avec les groupements d'achats? Comment les rabais sont-ils alloués et ces dispositions interferent-elles avec le mode optimal de réalisation des contrats? Comment les coûts et les profits des groupements d'achats sont-ils gérés? Quelle reconnaissance accorde-t-on aux sociétés pharmaceutiques qui ont mis en valeur le service à la clientèle, évité les ruptures de stock et maintenu des principes de gestion de la qualité?

Les pharmaciens doivent quantifier les répercussions des pénuries et des ruptures de stock des fournisseurs. Les pénuries de médicaments sont devenues un problème exigeant qui nécessite souvent l'intervention d'un technicien en pharmacie à temps plein (ou d'un pharmacien dans les établissements plus petits) pour trouver une source d'approvisionnement de rechange pour les produits en rupture de stock. Les gestionnaires dans de nombreux hôpitaux dépensent maintenant l'équivalent du salaire d'un technicien à temps plein (environ $50000 \$$ par année) pour s'occuper d'un problème externe d'approvisionnement. Une extrapolation rapide de cette figure de cas aux quelque 400 hôpitaux du Canada porte à croire que cette utilisation de ressources humaines se traduit par un fardeau de 20000000 \$ pour le système de soins de santé, touchant plus particulièrement les pharmacies hospitalières.

Comme Lybecker et Esmail l'ont décrit, "There are risks to drug bulk-purchasing agreements ${ }^{* 5}$ ". Alors que le désir des gouvernements provinciaux de réduire les coûts en médicaments est compréhensible, la question demeure, mais à quel prix? Lybecker et Esmail poursuivent en disant que " therés no free lunch: implementation of bulk-purchasing agreements and their interaction with other cost containment initiatives ... may negatively impact patients, and ultimately prevent the initiative from reducing overall expenditures $\dagger^{5}$ ”. Ils notent également que l'expérience de la Nouvelle-Zélande avec des mesures de maîtrise des coûts similaires " resulted in poorer care for some patients including increased prevalence of uncontrolled blood pressure, deteriorated lipid control, and worsened cardiovascular health $\ddagger$ " et que "Bulk purchasing agreements may also result in monopolies or a limited number of drug suppliers $\S^{5}$ ».

La pharmacie hospitalière a connu une évolution spectaculaire ces derniers dix ans en termes d'optimisation des politiques de distribution des médicaments et de collaboration avec les patients pour prendre en charge leurs traitements médicamenteux. Ces avancées sont maintenant menacées par le besoin d'allouer des ressources considérables pour composer avec les pénuries de médicaments. Au même moment, le rôle de la pharmacie dans la gestion de la chaîne d'approvisionnement en médicaments est passé en grande partie aux mains des entreprises d'achats groupés qui, souvent, ne sont pas en mesure de comprendre et d'apprécier pleinement les lacunes dans les contrats d'approvisionnement qu'ils négocient « en notre nom ". Le temps est venu pour les pharmacies de reprendre une partie des rôles qu'elles ont déjà joués dans la gestion de la chaîne d'approvisionnement en médicaments, afin d'assumer la responsabilité fondamentale de veiller à ce que les patients reçoivent un traitement médicamenteux optimal.

[Traduction par l'éditeur]

\section{Références}

1. Lignes directrices sur l'emballage et l'étiquetage des médicaments à l'intention des fabricants. Ottawa $(\mathrm{ON})$ : Société canadienne des pharmaciens d'hôpitaux; 2001.

2. Sellinger D. Pénuries de médicaments : le sondage a parlé... J Can Pharm Hosp. 2013;66(2):147.

3. Niccolai C, Skledar S. Updating smart pump libraries during shortages. Pharm Purch Prod. 2012;9(4):8. Publié au www.pppmag.com/article/1094/ April_2012/Updating_Smart_Pump_Libraries_during_Shortages/

4. Vaillancourt R. Pénuries de médicaments : Que peuvent faire les pharmaciens d'hôpitaux? [éditorial]. J Can Pharm Hosp. 2012;65(3):178-9.

\footnotetext{
* [Traduction] Il y a des risques associés aux ententes d'achat de médicaments en gros

$\dagger$ [Traduction] [...] il n'y a rien de gratuit : les ententes d'achat en gros et leur interaction avec d'autres mesures de contrôle des coûts [...] peuvent avoir une incidence négative sur les patients et au bout du compte empêcher les mesures d'atteindre leur objectif de réduction des dépenses totales.

‡[Traduction] [...] a entrainé une détérioration des soins de santé pour certains patients, se traduisant entre autres par une augmentation des cas d'hypertension non contrôlée, de dyslipidémie non maittrisée et de dégradation de la santé cardiovasculaire.

$\$$ [Traduction] Les ententes d'achat en gros peuvent aussi favoriser l'émergence de monopoles ou d'un nombre restreint de fournisseurs de médicaments.
} 
5. Lybecker KM, Esmail N. There are risks to drug bulk-purchasing agreements. Guelph Mercury 2013 May 31. Publié au www.guelphmercury.com/opinionstory/3249570-there-are-risks-to-drug-bulk-purchasing-agreements/. Consulté le 23 octobre 2013.

James L Mann, M. Sc. Pharm., FCSHP, a fait carrière exclusivement dans le domaine de la pharmacie hospitalière, plus particulièrement en qualité de directeur du service de pharmacie de plusieurs établissements. Il a été un membre actif de diverses associations de pharmacie et il a mené comme consultant un examen opérationnel du service de pharmacie dans plus de 40 hôpitaux canadiens. II a pendant ce temps également enseigné à temps partiel à des étudiants de premier cycle et il est maintenant membre du corps professoral à la retraite de la Faculté de pharmacie Leslie Dan de I'Université de Toronto, à Toronto, en Ontario. II a aussi été lauréat du Prix pour service distingué de la Société canadienne des pharmaciens d'hôpitaux en 1995.

Intérêts concurrents : aucun déclaré.

\section{Adresse de correspondance :}

James L Mann

Courriel : 7t2442@gmail.com
Cet article est l'un d'une série spéciale d'éditoriaux, sous la coordination de William McLean, Pharm. D., FASHP, FCCP, FCSHP, et du Comité de rédaction du $J C P H$, rédigés par des récipiendaires du Prix pour service distingué de la SCPH qui sont invités à commenter une question d'actualité pour la profession. L'éditorialiste invité doit fournir un bref point de vue sur l'évolution de cet aspect particulier au cours de sa carrière et commenter sa vision de l'évolution future de cet aspect. Par conséquent, l'éditorialiste invité combinera une perspective historique et future de même que des éléments de réflexion. 